\title{
The Effect of Collagen Activation on Platelet Rich Plasma for Proliferation of Periodontal Ligament Fibroblasts
}

\author{
Pati Tangsupati, Kwartarini Murdiastuti* \\ Department of Periodontology, Faculty of Dentistry, Universitas Gadjah Mada, Jl. Denta 1, Sinduadi, Yogyakarta, Indonesia \\ *Corresponding author. E-mail: kmurdiastuti@ugm.ac.id
}

Received date:Jan 25, 2018; Revised date: Jul 31, 2018; Accepted date: Aug 3, 2018

\section{Abstract}

$\mathrm{B}$ ACKGROUND: Regenerative procedure in periodontal surgery aims to improve the structure and function of the periodontium to be strong enough to support the teeth. Growth factor is essential in the process of tissue regeneration; it can be generated from the activation of Platelet-Rich Plasma (PRP). In this study, collagen was used as PRP activator. PRP release growth factor from the granules when activated. The aim of this study was to evaluate the effect of collagen on PRP activation and the collagen-activated PRP storage to fibroblast proliferation of periodontal ligament (PDL).

METHODS: Fibroblasts of PDL were obtained from extracted premolar. PRP were obtained from $100 \mathrm{~mL}$ of blood donors using double centrifugation methods. PRP were activated by collagen and subsequently incubated for 24, 48, 72 and 168 hours; after which the lysate was taken.
Fibroblasts were divided into 7 groups, which consisted of one unstimulated group as negative control, one group stimulated by PRP lysate, and five groups stimulated with PRP-collagen lysates that had been incubated for 24, 48, 72 and 168 hours. MTT assay was then performed after 1 and 3 days.

RESULTS: The proliferation rate of fibroblasts group stimulated by PRP-collagen lysate was higher than the group stimulated by PRP. The storage of collagen-activated PRP for 7 days at $4{ }^{\circ} \mathrm{C}$ could increase the proliferation rate.

CONCLUSION: The activation of collagen on PRP and the stored collaged-activated PRP could increase the fibroblasts proliferation rate of PDL.

KEYWORDS: collagen, platelet-rich plasma, fibroblasts proliferation

Indones Biomed J. 2018; 10(3): 278-83

\section{Introduction}

Platelet-Rich Plasma (PRP) is a high concentrate of autologous platelet obtained by centrifugation of autologous blood. PRP may release several types of growth factors such as platelet-derived growth factor (PDGF), transforming growth factor (TGF)- $\beta$, insulin growth factor (IGF), vascular endothelial growth factor (VEGF), platelet-derived endothelial cells growth factor (PDECGF), platelet-derived angiogenesis factor (PDAF) and fibroblasts growth factor (FGF).(1) PRP could increase the proliferation of fibroblasts and osteoblasts when used in a certain concentration range. (2) PRP on fibroblasts could stimulate adhesion and cell spreading on fibronectin matrix (3), as well as increasing in actin-enrich cellular extensions. It also promotes cell migration and invasion through the basement of matrix. The trend of fibroblasts proliferation rate has been observed in previous studies, and the results showed the longer the duration of stimulation of fibroblasts, the higher the proliferation rate that has been achieved. $(1,4)$

Platelet will release the content of granules when they are activated. Activation of platelet can occur when the adhesion molecules are exposed due to endothelial injury such as von Willebrand factor, collagen, fibronectin and laminin. Platelets can also be activated by physiological agonists such as thrombin, ADP, collagen, thromboxane A2, epinephrine and others.(5) 
The amount of platelet and growth factor obtained is affected by the method used in the preparation of PRP. The growth factor obtained affects the clinical outcomes.(2) PRP preparation processed with the addition of activators has been studied. Activation of PRP with thrombin showed a very fast release of growth factors while activation with collagen showed sustained release in a relatively same amount of time from day 1, 3, to day 7. Previous studies showed deficiency of bovine thrombin usage by the emergence of hypersensitivity reactions, impaired viability and altered migration of fibroblasts, decreased activity of FGF, and decreased clot strength.(6) Results of in vitro studies demonstrated the use of collagen is a safe and effective alternative, in addition to stimulating the release of growth factors, it also increase the consistency of PRP with gelation process.(7) Growth factors released from PRP activated by collagen include PDGF and TGF- $\beta$ which are potent stimulators for fibroblasts in cell migration, mitogenesis, proliferation and in matrix synthesis, this is important in wound healing. $(7,8)$

Collagen-based biomaterial has now become the most important material in the field of tissue engineering and regeneration because of its high biocompatibility and low immunogenicity. Collagen can also be combined with other molecules in the field of medical applications. Sources of collagen are also vary, but the most widely used in clinical applications is collagen derived from mammals such bovine, while collagen derived from rat tails are generally used in in vitro research. One of the solid forms of collagen is an absorbable collagen sponge (ACS), this biomaterial is used in dentistry as a hemostat or as scaffold in tissue regeneration process. In other research, collagen was used as a carrier recombinant human bone morphogenetic protein 2 (rhBMP-2) critical size injuries in red calf.(9) The results showed an increase in the bone formation. Recent research was using a collagen sponge as a carrier of PRP in treating gingival recession class I and II.(10) The results showed the addition of keratinized gingiva and an increase of clinical attachment level. The aimed of this study was to evaluate benefits of collagen in PRP activation and the collagen-activated PRP storage to fibroblast proliferation of periodontal ligament (PDL).

\section{Methods}

This research was an experimental laboratory and approved by ethics commite of the Faculty of Dentistry, Universitas Gadjah Mada Indonesia (No.420/KKEP/FKG-UGM/
$\mathrm{EC} / 2013)$. The following variables were: variable of effect was the kind of treatments (PRP, collagen-activated PRP), and observation time; the affected variable was proliferation of PDL fibroblasts. The object of research was primary PDL fibroblasts of the extracted premolars for orthodontic treatment. Collagen was obtained from rat tail (RT) collagen and collacure sponge collagen. PRP was obtained from blood centrifugation with double centrifugation method.For $24,48,72,168$ hours, it was activated by RT collagen and for 24 hours by collacure. Then they were incubated and observed after 1 and 3 days

\section{PRP Preparation}

Ten $\mathrm{mL}$ of blood was taken from human probandus and it was put into a tube containing $1 \mathrm{~mL}$ of $3.8 \%$ sodium citrate, following that, it was mixed slowly in order to make a homogenous mixture. The tube was centrifuged for 10 minutes in $1200 \mathrm{rpm}$ rotation speed. The centrifugation produced two layer, the top layer was contained platelet-poor plasma (PPP) and the bottom layer was contained red blood cells PPP layer was taken using a long canula + water-intake canula was included into tubes without anticoagulant. The tube was centrifuged for 10 minutes at $3500 \mathrm{rpm}$ rotation speed. This second centrifugation produced two layers, and then it was separated from PPP with isolation kit.(7)

\section{PRP Activation}

Activation of PRP was obtained by mixing the supernatant PRP and collagen, the volume ratio was 1:15.

\section{Fibroblasts's Culture}

PDL cells taken from the root surface of teeth using a blade \#15. The ligament was taken in the middle third of tooth root, ligament was cut and then cultured using Dubelco's Modification of Eagle's Medium (DMEM), added a 10\% of fetal bovine serum (FBS) supplement, $2 \%$ of penicillinstreptomycin, $0.5 \%$ fungi zone and subsequently put into incubator in temperature of $37^{\circ} \mathrm{C}$.(6)

PDL fibroblast was cultured in a media in microplate 24 wells and consisted of 7 groups, which are unstimulated fibroblasts or negative control (NC), fibroblasts stimulated by PRP lysate, fibroblasts stimulated by PRP + collagen RT lysates and incubated for 24 hours (T1), fibroblasts stimulated by PRP + collagen RT lysates and incubated for 48 hours (T2), fibroblasts stimulated by PRP + collagen RT lysates and incubated for 72 hours (T3), fibroblasts stimulated by PRP + collagen RT lysates and incubated for 168 hours (T4), and fibroblasts stimulated by PRP + collacure lysates incubated for 24 hours (T5). Each group consisted of 10 
wells and were incubated at $37^{\circ} \mathrm{C}$. Fibroblast proliferation of each group was measured by 3-(4,5-dimethylthiazol-2yl)-2,5-diphenyltetrazolium bromide (MTT) assay on day 1 and 3 (observation times). Data were analyzed using Twoway ANOVA, followed by least significant differenc (LSD) test with a confidence level of $95 \%$.

\section{Results}

Data in this research were taken from spectrophotometry plate with a wave length of $570 \mathrm{~nm}$. The mean of fibroblast proliferation rate and the standard deviation based on observation times (day 1 and 3 ) in each group is presented in Table 1 below.

Table 1. The mean and standard deviation of the periodontal ligament fibroblast proliferation rate.

\begin{tabular}{lcc}
\hline \multirow{2}{*}{ Groups } & \multicolumn{2}{c}{ Observation time } \\
\cline { 2 - 3 } & $\begin{array}{c}\text { Day 1 } \\
(\mathbf{n}=\mathbf{4 2})\end{array}$ & $\begin{array}{c}\text { Day 3 } \\
(\mathbf{n}=\mathbf{4 2})\end{array}$ \\
\hline PRP & $0.2043 \pm 0.0354$ & $0.3773 \pm 0.0539$ \\
NC & $0.1055 \pm 0.0082$ & $0.2323 \pm 0.0459$ \\
T1 & $0.4365 \pm 0.1604$ & $0.4628 \pm 0.0574$ \\
T2 & $0.4363 \pm 0.0815$ & $0.5375 \pm 0.0759$ \\
T3 & $0.3810 \pm 0.0318$ & $0.4885 \pm 0.0533$ \\
T4 & $0.3767 \pm 0.0791$ & $0.4898 \pm 0.0378$ \\
T5 & $0.4138 \pm 0.0647$ & $0.4972 \pm 0.0629$ \\
\hline
\end{tabular}

Table 1 showed that the mean of PDL fibroblast proliferation was lowest in the $\mathrm{NC}$ group with a mean and standard deviation of $0.1055 \pm 0.0082$ and $0.2323 \pm 0.0459$ on day 1 and 3 respectively. In each group, average fibroblast proliferaton rate on day 3 was higher than that of day 1 .

Figure 1 showed that the lowest mean and standard deviation of fibroblast proliferation rate were same in $\mathrm{NC}$ in both groups whereas the highest one was seen in a group that incubated PRP-activated RT collagen at 24 hours of incubation in day 1 and at 48 hours of incubation in day 3.

Normality and homogeneity tests showed a normally distributed and homogen data $(p>0.05)$ therefore the statistical analysis was continued using two-way ANOVA parametric test in order to find out whether there were differences among PDL fibroblast applied by PRP, PRP activateed collagen and a group which was not applied by PRP activated collagen in different observation times of storage (day 1 and 3). The result is presented in Table 2. It implied that there was an effect of group and time of observation to the proliferation cells of fibroblast in PDL but no effect on interaction between group and time.

Table 2. Result of two-way ANOVA test of periodontal ligament fibroblast proliferation.

\begin{tabular}{lccc}
\hline & $\begin{array}{c}\text { Deegre of } \\
\text { Freedom }\end{array}$ & F & $\boldsymbol{p}$ \\
\hline Group & 6 & 39.719 & $0.000^{*}$ \\
Time & 1 & 40.88 & $0.000^{*}$ \\
Group x Time & 6 & 1.672 & 0.141 \\
\hline
\end{tabular}

*: significant $p$-value

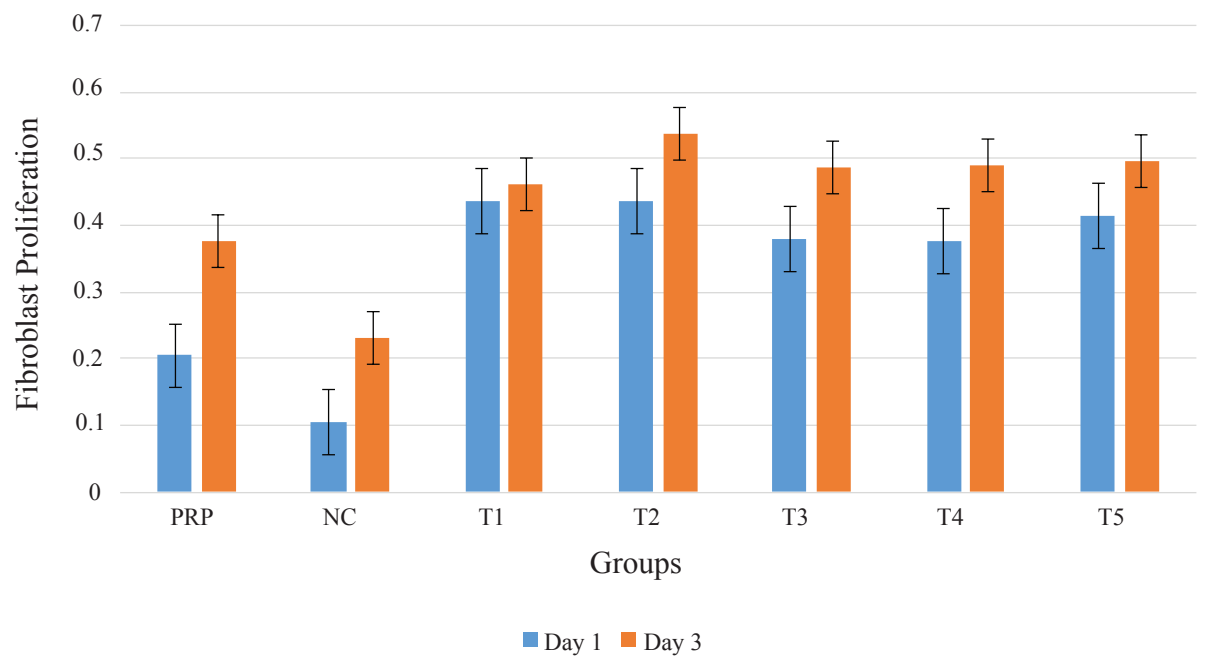

Figure 1. Graph of fibroblast proliferation rate on day 1 and 3. PRP: Fibroblasts + 24-hour incubation of PRP lysates; NC: Fibroblasts without stimulation; T1: Fibroblast + PRP + RT collagen lysate, 24 hours incubation; T2: Fibroblasts + PRP + RT collagen lysate, 48 hours incubation; T3: Fibroblasts + PRP + RT collagen lysate, 72 hours incubation; T4: Fibroblasts + PRP + RT collagen lysate, 168 hours incubation; T5: Fibroblasts + PRP + RT collacure lysate, 48 hours incubation. 
The result in Table 2 above was subsequently continued with Post-Hoc LSD test to see the difference of proliferation among groups and the observation times. The LSD test is shown in Table 3.

Table 3 indicated that in day 1 of observation time, there were significant differences of PDL fibroblast proliferation rate between group of PRP with negative control, PRP activated RT collagen in all incubations, and between negative control with all other groups. There were no significant differences among PRP activated collagen with different incubation times.

Table 3. LSD test of periodontal ligament fibroblas proliferation among groups on day 1 .

\begin{tabular}{lcccccc}
\hline & NC & T1 & T2 & T3 & T4 & T5 \\
\hline PRP & $0.040^{*}$ & $0.000 *$ & $0.000^{*}$ & $0.001 *$ & $0.001 *$ & $0.000^{*}$ \\
NC & & $0.000 *$ & $0.000 *$ & $0.000^{*}$ & $0.000^{*}$ & $0.000^{*}$ \\
T1 & & & 0.997 & 0.239 & 0.205 & 0.628 \\
T2 & & & & 0.241 & 0.207 & 0.631 \\
T3 & & & & & 0.926 & 0.484 \\
T4 & & & & & & 0.428 \\
\hline
\end{tabular}

*: significant $p$-value

Table 4 implied that in day 3 of observation time, there were significant differences of PDL fibroblast proliferation between PRP with negative control groups, PRP-activated RT collagen in all incubation times, and between negative control groups with all other groups. There were no significant differences among each PRP activated collagen group with different incubation times, except between PRP-activated RT collagen group with 24 and 48 hours of incubation.

Table 4. LSD test of periodontal ligament fibroblast proliferation among all groups in day 3 .

\begin{tabular}{lcccccc}
\hline & NC & T1 & T2 & T3 & T4 & T5 \\
\hline PRP & $0.000^{*}$ & $0.013^{*}$ & $0.000^{*}$ & $0.002^{*}$ & $0.001 *$ & $0.001 *$ \\
NC & & $0.000^{*}$ & $0.000^{*}$ & $0.000^{*}$ & $0.000^{*}$ & $0.000^{*}$ \\
T1 & & & $0.028^{*}$ & 0.436 & 0.413 & 0.3 \\
T2 & & & & 0.142 & 0.153 & 0.224 \\
T3 & & & & & 0.968 & 0.207 \\
T4 & & & & & & 0.823 \\
\hline
\end{tabular}

*: significant $p$-value
Because there was an effect of observation time to the proliferation cells of fibroblast in PDL thus both groups of observation times was then analyzed using independent sample T-test and the result is presented in Table 5.

Table 5 showed that there were significant differences of PDL fibroblast proliferation between day 1 and 3 of observation times in all treatment groups. However, there were no significant differences in groups that were applied using PRP-activated RT collagen with 24 hour-incubation.

Table 5. Independent sample T-test of periodontal ligament fibroblast proliferation between each observed times.

\begin{tabular}{lc}
\hline Group & $\boldsymbol{p}$-value \\
\hline PRP & $0.000^{*}$ \\
NC & $0.000^{*}$ \\
T1 & 0.713 \\
T2 & $0.050^{*}$ \\
T3 & $0.002^{*}$ \\
T4 & $0.010^{*}$ \\
T5 & $0.470^{*}$ \\
\hline *: significant $p$-value
\end{tabular}

\section{Discussion}

The effect of PRP to the proliferation rate of PDL fibroblast can be seen on Table 1 with the lowest mean was observed in the negative control group. Data analyzed using PostHoc LSD test is shown on Table 3 and 4. On day 1 and 3 , there were significant differences between negative control group (without PRP) compared to other groups of PRP. It means the proliferation rate of PDL fibroblast in the group stimulated by PRP was higher than that of without PRP. Therefore, PRP could increase fibroblast proliferation with observation time on day 1 and 3. This result was in line with research by Graziani, et al., that stated PRP can increase proliferation rate within 24 and 72 hours in in vitro research.(1) Yilmaz, et al., also declared that PRP is capable to regulate biologic events such as cell proliferation, cell attachment, cell migration and extracellular matrix synthesis. (11) This may happen because PRP is supernatant with high concentration platelet that can produce various growth factors if granules in platelet are activated. Growth factor is a very important signaling molecule in various cellular processes. Each growth factor has regulatory function on cellular level and is seen in the tissue regeneration process. 
Growth factor is produced in platelet granulation process including TGF- $\beta 1$ to control proliferation and differentiation of various cell types; PDGF-B to stimulate proliferation and disrupt apoptosis; FGF inducted angiogenesis and fibroblast proliferation; IGF acts as a mediator for other growth factors and as chemotactic character on fibroblast of PDL; VEGF acts in angiogenesis process. The research proved that PRP releases TGF- $\beta 1$ and PDGF-AB 3 and 2.5 times that of whole blood respectively.(6)

Effect of collagen activation on PRP to fibroblast proliferation rate and the figure of PDLfibroblast proliferation on observation day 1 and 3 can be observed on Table 3 and 4. There were significant differences between PRP group (inactive PRP) compared to other groups of activated PRP. Those differences showed that adding collagen increased the proliferation of PDL fibroblast on day 1 and 3. It meant that the proliferation of PDL fibroblast on all groups of activated PRP with different incubation times were higher than that of the inactive PRP group. Therefore, the activation of PRP using any collagen (RT collagen or collacure sponge from bovine's Achilles) could increase fibroblast proliferation with observation times of day 1 and 3 . This may cause the growth factor to be released from platelet granules through molecular interaction process when platelet is activated. Enhancing platelet activation process is important in tissue regeneration process.

Collagen was used, in this research, to activate platelet granulation process. Collagen is a triple helix protein molecule with naturally involved in cascade aggregation and platelet degranulation within a body. $(5,12)$ Platelet activation is a complex process that involved series of feedbacks and crosstalk between different pathways. Signaling pathway in various specific receptors of platelet simultaneously stimulated platelet form changes, growth factor secretion and other molecules from granule and at the end inducted inside-out signal process that activated $\alpha 2 b \beta 3$ integrin bond with its ligand. This bond was then caused platelet adhesion and aggression as well as triggered outside-in signal thus caused the secretion of granule content, stabilize adhesion, aggression and platelet clot. Platelet activation process inducted by collagen started with collagen bond with glycoprotein (GP) VI receptor, which connected to $\mathrm{FcR} \gamma$, this bond inducted few intracellular signal series and activated integrin $\alpha 2 \beta 1$ which later bonded with other collagen molecules, integrin $\alpha 2 b \beta 3$ as receptor of fibrinogen, integrin $\alpha v \beta 3$ receptor of vitronectin and integrin $\alpha 5 \beta 1$ receptor of fibronectin. Collagen bond with 2 prominent receptors then inducted intercellular signal cascade which lead to calcium release and activation of kynase C (PKC) protein; those were responsible to platelet activation towards series of those signals, platelet response such as aggregation was through exocytosis process. $(13,14)$ The pattern of growth factor released from platelet granules can be different depending on the activator used. Harrison, et al., found PRP activated by collagen released growth factor continuously for 7 days and released more TGF $\beta-1$ in 7 days observation compared to the activation with thrombin.(6) While the total PDGF-AB and VEGF released was relatively same on either PRP activated by thrombin or by collagen in 7 days. TGF $\beta-1$ is a growth factor with a major role in the process of fibroblast proliferation.

Table 3 and 4 showed that there was no effect of collagen incubation time on PRP to the fibroblast proliferation. Activation time in this research was equal with PRP storage time. PRP was activated by collagen and kept for 24 hours (T1), 48 hours (T2), 72 hours (T3) and 168 hours (T4). After the activation, PRP lysate was take and applied PDL culture cell. It was meant to see whether activation or incubation time or storage time in $4^{\circ} \mathrm{C}$ temperature (collagen activated PRP) could be affected or not to stimulate proliferation of PDL fibroblast. The result of Post-Hoc LSD showed comparison among group T1, T2, T3 and T4 observation on day 1 was not significantly different $(p>0.05)$. The similar pattern showed on observation day 3 , the comparison among groups (T1, T3, and T4) did not show any significant difference $(p>0.05)$. This proved that PRP lysate activated with collagen and incubated for 24,72 and 168 hours did not show differences or give the meaning was same or equal in stimulating fibroblast proliferation. This showed that numbers on growth factors in PRP lysate after incubation on the period of time had the same value to stimulate proliferation of periodontal fibroblast. The results of this study were in accordance with the conclusions obtained by Poerty, et al., that there was no different between directly and after 24 hours of long-term activation PRP with collagen on growth factors, especially fibroblast growth factor-2 (FGF-2).(16)

Table 5 showed there was a significant difference between proliferation figure on observation on day 1 and 3 in all groups with $p<0.05$. It explained that the process of proliferation of PDL fibroblast in this research had a significant increase every day. The result is in line with prior research in which the figure of fibroblast proliferation increased by the time or time dependent. $(1,3,15)$ An exception to group $\mathrm{T} 1$ also had an increase in the mean of proliferation rate, however, using independent sample T-test, the result was not significant. This could be caused by technical factors in laboratory during incubation process 
and treatment using MTT assay. Undissolved Formosan salt by reagent stopper (DMSO) could decrease the score of color density by plate reader that caused absorbency figure to be invalid.

PDL fibroblasts have the most important role in the regeneration of periodontal tissue because of its ability to regenerate soft tissue and hard tissue. Therefore, this study used it as a representation of the periodontal tissues. PRP is a source of growth factors that can stimulate cellular activities, such as cell proliferation. PRP that is activated by collagen could also decrease the PRP clot retraction. PRP and collagen had shown to increase proliferation up to 4 times higher. Increased fibroblast proliferation will accelerate regeneration, thus may also clinically accelerate the healing process and improve clinical outcomes of a treatment. According to the author's knowledge, there has never been a study that examined the PRP of collagen activation in other cells.

\section{Conclusion}

In conclusion, the result of this study indicate that collagen activation on PRP could increase periodontal fibroblast proliferation. Incubation time of collagen activation on PRP (activated PRP storage time) was not affecting the proliferation of PDL fibroblast, in the other hand, observation time affected the PDL fibroblast. Collacure sponge collagen had the same efficacy with rat tail collagen in inducing PDL fibroblast proliferation. We suggest for next research to examine the effect of collagen addition on PRP at the in vivo setting, to see its effect on tissue regeneration.

\section{References}

1. Graziani F, Cei S, Ducci F, Gabriele M, Ivanovski S, Tonetti M. The in vitro effect of different PRP concentration on osteoblasts and fibroblasts. Clin. Oral Impl. Res. 2006; 17: 212-9.
2. Careces M., Hidalgo R, Sanz A., Martinez J. Effect of plateletrich plasma on cell adhesion, cell migration, and myofibroblastic differentiation in human gingival fibroblast. J Periodontol. 2008; 79: 714-20.

3. Manoranjan SJ, Faizuddin M, Hemalatha M, Ranganath V. The effect of platelet derived growth factor- $\mathrm{AB}$ on periodontal ligament fibroblasts: an in vitro study. J Indian Soc Periodontal. 2012; 16: 49-53.

4. Park HB, Yang JH, Chung KH. Characterization of the cytokine profile of platelet rich plasma (PRP) and PRP-induced cell proliferation and migration: Upregulation of matrix metalloproteinase-1 and -9 in HaCaT cells. Korean J Hematol. 2011; 46; 265-72.

5. Sánchez-González DJ, Méndez-Bolaina E, Trejo-Bahena NI. Plateletrich plasma peptides: key for regeneration. Int J Pept. 2012; 2012:5 32519. doi: $10.1155 / 2012 / 532519$.

6. Harrison S, Vavken P, Kevy S, Jacobson M, Zurakowski D, Murray MM. Platelet activation by collagen provides sustained release of anabolic cytokines. Am J Sports Med. 2011; 39: 729-34.

7. Fufa D, Shealy B, Jacobson M, Kevy S, Murray MM. Activation of platelet-rich plasma using soluble type I collagen. J Oral Maxillofac Surg. 2008; 66: 684-90.

8. Anusakthien O, Webb SA, Jin QM, Giannobile WV. Platelet-derived growth factor gene delivery stimulates ex vivo gingival repair. Tissue Eng. 2003; 9: 745-56.

9. Mariner DP, Wudel JM, Miller DE. Synthetic hydrogel scaffold is an effective vehicle for delivery of INFUSE (rhBMP-2) to criticalsized calvaria bone defects in rats. J Orthop Res. 2013; 31: 401-16.

10. Naik AR, Ramesh AV, Dwarkanath CD. Use of autologous platelet rich plasma to treat gingival recession in esthetic periodontal surgery. J Indian Soc Periodontol. 2013;17: 345-53.

11. Yilmaz A, Cakar G, Ipci SD. Platelet rich plasma in reconstructive periodontal theory. In: Progress in Molecular and Environmental Bioengineering. London: IntechOpen; 2011. p.269-83.

12. Bareil RP, Gauvan R, Berthod F. Collagen-based biomaterials for tissue engineering applications. Materials. 2010; 3: 1863-87.

13. Li Z, Delaney MK, O’Brien KA, Du X. Signaling during platelet adhesion and activation. Artioscler Thromb Vasc Biol. 2010: 30: 2341-9.

14. Jackson EC, Ortar G, McNicol A. The effect of an inhibitor of diglyceride lipase on collagen-induced platelet activation. J Pharmacol Exp Ther. 2013; 347: 582-8.

15. Kim DH, Je YJ, Kim CD, Lee YH, Seo YJ, Lee JH, Lee Y. Can platelet-rich plasma be used for skin rejuvenation? Evaluation of effect of platelet-rich plasma on human dermal fibroblast. Ann Dermatol. 2011; 23: 424-31.

16. Poerty AD, Murdiastuti K, Sudibyo. Pengaruh Lama Aktivasi Platelet-Rich Plasma dengan Kolagen terhadap Kadar Fibroblast Growth Factor-2 Cairan Sulkus Gingiva pada Terapi Resesi Gingiva [Thesis]. Yogyakarta: Universtas Gadjah Mada; 2016. 